Editorial

\section{Erreichtes und künftige Ziele}

\section{B. Kornhuber}

Das 7jährige Bestehen der Gesellschaft für Pädiatrische Onkologie ist Anlaß, bislang Erreichtes und Anstehendes zu überdenken. Der Impuls zur Gründung der Gesellschaft ging von Pä diatern aus, die in der Arbeitsgemeinschaft für Leukämieforschurg und -behandlung im Kindesalter e.V. 7 Jahıre lang auf dem Gebiet der Pädiatrischen Onkologie in gemeinsamer Arbeit Erfahrung gesammelt hatten. Der Grund für die Gesellschaftsgiündung war der Wunsch, einen weiteren Rahmen zu finden, um die anderen an Diagnostik und Therapie kindlicher Malignome beteiligten Disziplinen besser zu inkorporieren. Dies ist gelungen.

Das Forum für eine regelmäßige Diskussion und Information ist in den halbjährlich stattfindenden Tagung gegeben. Hinzukommen als Publikationsorgan die jährlich erscheinenden ,Ergebnisse der Pädiatrischen Onkologie “. Diese dienen dem Bericht über die vorausgegangenen Tagungen, sollten aber auch Raum für Publikationen bieten, die im Rahmen einer Tagung aus thematischen oder zeitlichen Gründen nicht unterzubringen warerı. Die für gemeinsame Therapiestudien notwendigen Voraussetzungen sind geschaffen worden:

- das (pathologisch-anatomische) Kindertumorregister in Kiel und die

- Zentrale Dokumeritation (Datensammlung und -verarbeitung) in Mainz zur Erfassung aller Malignome im Kindesalter unter Einschluß der Leukämien

Die Möglichkeiten der einheitlichen Klassifikation und des Gradings (Subklassifikation) sind ebenso unabdingbar wie die Aufarbeitung der klinischen Daten zur Durchführung der Therapiestudien und zur Planung künftiger Projek te. Darüber hinaus stehen jedem Kliniker die gespeicherten Daten der eigenen Patienten zur Verfügung. Das Ange bot des Mainzer Institutes reicht bis hin zur Patien teneinbestellung bei längerfristigen Terminen.
Klin. Pädiat. 192 (1980) 95

(C) F. Enke Verlag Stuttgart
Für Mitglieder der Gesellschaft, die an der Datengewinnung und -verarbeitung interessiert sind, besteht die Möglichkeit, zu Lasten einer Stelle, die im Mainzer Institut vorgehalten wird, sich einige Wochen zu informieren. Dieser enge Austausch soll für Klinik und Datenverarbeitung in gleicher Weise von Nutzen sein.

Die Dokumentation der Gesellschaft wurde in Einklang gebracht mit der Basisdokumentation der Arbeitsgemeinschaft Deutscher Tumorzentren (ADT). Doppelarbeit durch unterschiedliche Dokumentationsbögen wurde so für Mitglieder von Tumorzentren vermieden.

Besonders intensiv waren die Bemühungen der Gesellschaft, die personelle Situation in pädiatrisch-onkologischen Einheiten zu verbessern. Die Qualität der Arbeit - Diagnostik, Therapie, Dokumentation - ist von einer ausreichenden Personalausstattung abhängig. So wurde durch unsere Initiative von der ADT durch einen Ausschuß der Personalbedarf für onkologische Ambulanzen und Stationen in Tumorzentren erarbeitet. Die Richtdaten der GPO fanden dabei Berücksichtigung. Der besonderen Intensität und der weitergehenden Zentralisation der Behandlung im pädiatrischen Bereich wurde in diesem Zusammenhang Rechnung getragen. Mancherorts haben unsere Bemühungen erste Erfolge gehabt. Ein Personalzugang konnte erreicht werden.

Die Bereitschaft zur Kooperation ist in den letzten Jahren gewachsen. Wünsche sind aber auch hier noch offen. Für alle größeren Tumorentitäten müssen prospek tive randomisierte Studien etabliert werden. Für derartige Studien soll die Möglichkeit der personellen Unterstïtzung geschaffen werden. Die einzelne Klinik kann so in die Lage versetzt werden, die aufwendige Arbeit innerhalb einer Studie zu übernehmen. Unsere klinische Arbeit wird in Rahmen prospek tiver Studien auswertbar und vergleichbar mit den Ergebnissen anderer Therapiegruppen. Es liegt an uns, die geschaffenen Mög. lichkeiten zu nu tzen. 